\title{
INTEGER COMPLEXITY: THE INTEGER DEFECT
}

\author{
HARRY ALTMAN
}

\begin{abstract}
Define $\|n\|$ to be the complexity of $n$, the smallest number of ones needed to write $n$ using an arbitrary combination of addition and multiplication. John Selfridge showed that $\|n\| \geq 3 \log _{3} n$ for all $n$, leading this author and Zelinsky to define the defect of $n, \delta(n)$, to be the difference $\|n\|-3 \log _{3} n$. Meanwhile, in the study of addition chains, it is common to consider $s(n)$, the number of small steps of $n$, defined as $\ell(n)-\left\lfloor\log _{2} n\right\rfloor$, an integer quantity. So here we analogously define $D(n)$, the integer defect of $n$, an integer version of $\delta(n)$ analogous to $s(n)$. Note that $D(n)$ is not the same as $\lceil\delta(n)\rceil$.

We show that $D(n)$ has additional meaning in terms of the defect wellordering considered in [3], in that $D(n)$ indicates which powers of $\omega$ the quantity $\delta(n)$ lies between when one restricts to $n$ with $\|n\|$ lying in a specified congruence class modulo 3 . We also determine all numbers $n$ with $D(n) \leq 1$, and use this to generalize a result of Rawsthorne 18 .
\end{abstract}

\section{INTRODUCTION}

The complexity of a natural number $n$, denoted $\|n\|$, is the least number of 1 's needed to write it using any combination of addition and multiplication, with the order of the operations specified using parentheses grouped in any legal nesting. For instance, $n=11$ has a complexity of 8 , since it can be written using 8 ones as

$$
11=(1+1+1)(1+1+1)+1+1,
$$

but not with any fewer than 8. This notion was implicitly introduced in 1953 by Kurt Mahler and Jan Popken [17, and was later popularized by Richard Guy [14, 15.

Integer complexity is approximately logarithmic; it satisfies the bounds

$$
3 \log _{3} n=\frac{3}{\log 3} \log n \leq\|n\| \leq \frac{3}{\log 2} \log n, \quad n>1 .
$$

The lower bound can be deduced from the results of Mahler and Popken, and was explicitly proved by John Selfridge [14]. It is attained with equality for $n=3^{k}$ for all $k \geq 1$. The upper bound can be obtained by writing $n$ in binary and finding a representation using Horner's algorithm. It is not sharp, and the constant $\frac{3}{\log 2}$ can be improved for large $n$ 22.

Based on the lower bound, this author and Zelinsky [ 6 introduced the notion of the defect of $n$, denoted $\delta(n)$, which is the difference $\|n\|-3 \log _{3} n$. Subsequent work [3] showed that the set of defects is in fact a well-ordered subset of the real line, with order type $\omega^{\omega}$.

However, it is worth considering the result of Selfridge in more detail:

Date: August 1, 2018. 
Theorem 1.1 (Selfridge). For any $k \geq 1$, let $E(k)$ be the largest number that can be made with $k$ ones, i.e., the largest $n$ with $\|n\| \leq k$. Then:

(1) If $k=1$, then $E(k)=1$.

(2) If $k \equiv 0(\bmod 3)$, then $E(k)=3^{k / 3}$.

(3) If $k \equiv 1(\bmod 3)$ and $k>1$, then $E(k)=4 \cdot 3^{(k-4) / 3}$.

(4) If $k \equiv 2(\bmod 3)$, then $E(k)=2 \cdot 3^{(k-2) / 3}$.

(This result is also a special case of the results of Mahler and Popken [17.). From this one can of course derive the lower bound $\|n\| \geq 3 \log _{3} n$, but what if one wanted an integer version? We make the following definition:

Definition 1.2. Given a natural number $n$, we define $L(n)$ to be the largest $k$ such that $E(k) \leq n$.

With this, we define:

Definition 1.3. For a natural number $n$, we define the integer defect of $n$, denoted $D(n)$, to be the difference $\|n\|-L(n)$.

Because of Theorem 1.1. $L(n)$ is quite easy to compute (see Proposition 3.8), and hence if one knows $\|n\|$ then $D(n)$ is also easy to compute. Note that while we consider $D(n)$ to be an integer analogue of $\delta(n)$, it is not in general equal to $\lceil\delta(n)\rceil$; see Theorem 3.12 for the precise relation. However it's not immediately obvious that $D(n)$ has any actual significance. In fact, however, the integer defect of a number tells you about its position in the well-ordering of defects.

Remark 1.4. $L(k)$ is not the best lower bound we can get from Theoerem 1.1 that would instead be the smallest $k$ such that $E(k) \geq n$, which we might denote $L^{\prime}(n)$. $\left(L^{\prime}(n)\right.$ could also be defined as the minimum of $\|m\|$ over all $m \geq n$.) For reasons that will become clear later, though, we will prefer to discuss $L$ rather than $L^{\prime}$. In any case, $L^{\prime}(n)=L(n)+1$ unless $n=E(k)$ for some $k$, in which case $L^{\prime}(n)=L(n)=k$, so one can easily convert any results expressed in the one formulation to the other. One could consider a similar $D^{\prime}(n)$ as well, but we will not do that either.

1.1. The sets $\mathscr{D}^{0}, \mathscr{D}^{1}$, and $\mathscr{D}^{2}$ and the main result. As has been noted above, if we define $\mathscr{D}$ to be the set of all defects, then as a subset of the real line this set is well-ordered and has order type $\omega^{\omega}$. However, more specific theorems are proved in [3. We will need the following definition:

Definition 1.5. If $a$ is a congruence class modulo 3, we define

$$
\mathscr{D}^{a}=\{\delta(n):\|n\| \equiv a \quad(\bmod 3), n \neq 1\} .
$$

Remark 1.6. The number $n=1$ is excluded from $\mathscr{D}^{1}$ because it is dissimilar to other numbers whose complexity is congruent to 1 modulo 3 . Unlike other numbers which are 1 modulo 3 , the number 1 cannot be written as $3 j+4$ for some $j \geq 0$, and so the largest number that can be made with a single 1 is simply 1 , rather than $4 \cdot 3^{j}$.

In fact the sets $\mathscr{D}^{a}$ for $a=0,1,2$ are disjoint, and so together with $\{1\}$ form a partition of $\mathscr{D}$.

Moreover in [3] it was proved:

Theorem 1.7. For $a=0,1,2$, the sets $\mathscr{D}^{a}$ are all well-ordered, each with order type $\omega^{\omega}$. 
It is these sets, the $\mathscr{D}^{a}$, that $D(n)$ will tell us about the position of $\delta(n)$ in. We show:

Theorem 1.8 (Main theorem). Let $n>1$ be a natural number Let $\zeta$ be the order type of $\mathscr{D}^{\|n\|} \cap[0, \delta(n))$. Then $D(n)$ is equal to the smallest $k$ such that $\zeta<\omega^{k}$.

As mentioned above, $D(n)$ is easy to compute, so this theorem gives an way to easily compute where around $\delta(n)$ falls in the ordering on $\mathscr{D}^{a}$.

We will also prove a version of this theorem for the stable integer defect; see Sections 1.4 and 3 .

It's worth comparing this theorem to what was already known. It was proved in 3 that the limit of the initial $\omega^{k}$ elements of $\mathscr{D}$ is equal to $k$. This raises the question - just what is the limit of the initial $\omega^{k}$ elements of $\mathscr{D}^{a}$ ? It was further shown in 3 ] that when $k \equiv a(\bmod 3)$ this limit is equal to $k$, but what about otherwise?

In this paper we will answer this question:

Theorem 1.9. The limit of the initial $\omega^{k}$ elements of $\mathscr{D}^{a}$ is equal to $k$ if $k-a \equiv 0$ $(\bmod 3)$; it is equal to $k+\delta(2)$ if $k-a \equiv 1(\bmod 3)$; and it is equal to $k+2 \delta(2)$ if $k-a \equiv 2(\bmod 3)$.

In fact, Theorem 1.9 will be used to prove Theorem 1.8. See Section 4 for more general statements. Further generalizations will appear in a future paper 5 .

1.2. Generalizing Rawsthorne's theorem. We know how to compute $E(k)$, the highest number of complexity at most $k$ (or exactly $k$ ), but what about the next highest? This question was answered by Daniel Rawsthorne 18 in 1989:

Theorem 1.10 (Rawsthorne). For any $k \geq 8$, the highest number of complexity at most $k$ other than $E(k)$ itself is $\frac{8}{9} E(k)$, and this number has complexity exactly $k$.

In this paper we generalize this result. First, a definition:

Definition 1.11. Given $r \geq 0$ and $k \geq 1$, we define $E_{r}(k)$ to be the $r$ 'th largest number of complexity at most $k$. We will 0 -index here, so that by definition $E_{0}(k)=$ $E(k)$, and Theorem 1.10 gives a formula for $E_{1}(k)$.

Then, with this, we show:

Theorem 1.12. Given $r \geq 0$, and a a congruence class modulo 3 , there exists $K_{r, a}>1$ and $h_{r, a} \in \mathbb{Q}$ such that for $k \geq K_{r, a}$ with $k \equiv a(\bmod 3)$, we have $E_{r}(k)=h_{r, a} E(k)$, and these $h_{r, a}$ and $K_{r, a}$ are as given by Tables 1, 2, and 3. Moreover, for such $r$ and $k$, we have $E_{r}(k)>E(k-1)$ and therefore $\left\|E_{r}(k)\right\|=k$ (and thus for such $r$ and $k, E_{r}(k)$ is not just the $r$ 'th largest number with complexity at most $k$, but the $r$ 'th largest number with complexity exactly $k$ ).

Note that Tables 1, 2, and 3 don't list the regular pattern in the $h_{r, a}$ until such point as $K_{r, a}$ also becomes regular; for tables based solely on $h_{r, a}$, see Tables 4, 5. and 6 .

What does Theorem 1.12 have to do with integer defect? Well, the numbers $h_{r, a} E(k)$ appearing in this theorem are almost exactly the numbers $n$ with $D(n) \leq$ 1; see Proposition 5.6 for a precise statement.

After all, by Theorem 1.8, the numbers $n$ with $D(n) \leq 1$ are precisely those $n$ whose $\delta(n)$ lie in the initial $\omega$ of $\mathscr{D}^{\|n\|}$. So if one fixes a particular $k$, then going 
TABLE 1 . Table of $h_{r}$ and $K_{r}$ for $k \equiv 0(\bmod 3)$.

\begin{tabular}{|c|c|c|}
\hline$r$ & $h_{r, 0}$ & $K_{r, 0}$ \\
\hline 0 & 1 & 3 \\
\hline 1 & $8 / 9$ & 6 \\
\hline 2 & $64 / 81$ & 12 \\
\hline 3 & $7 / 9$ & 12 \\
\hline 4 & $20 / 27$ & 12 \\
\hline 5 & $19 / 27$ & 12 \\
\hline 6 & $512 / 729$ & 18 \\
\hline 7 & $56 / 81$ & 18 \\
\hline 8 & $55 / 81$ & 18 \\
\hline 9 & $164 / 243$ & 18 \\
\hline 10 & $163 / 243$ & 18 \\
\hline$($ for $n \geq 6) \quad 2 n-1$ & $2 / 3+2 / 3^{n}$ & $3 n$ \\
\hline$($ for $n \geq 6) \quad 2 n$ & $2 / 3+1 / 3^{n}$ & $3 n$ \\
\hline
\end{tabular}

TABLE 2. Table of $h_{r}$ and $K_{r}$ for $k \equiv 2(\bmod 3)$.

\begin{tabular}{|rr|r|r|}
\hline & $r$ & $h_{r, 2}$ & $K_{r, 2}$ \\
\hline & 0 & 1 & 2 \\
& 1 & $8 / 9$ & 8 \\
& 2 & $5 / 6$ & 8 \\
& 3 & $64 / 81$ & 14 \\
& 4 & $7 / 9$ & 14 \\
& 5 & $20 / 27$ & 14 \\
& 6 & $13 / 18$ & 14 \\
& 7 & $19 / 27$ & 14 \\
& 8 & $512 / 729$ & 20 \\
& 9 & $56 / 81$ & 20 \\
& 10 & $37 / 54$ & 20 \\
& 11 & $55 / 81$ & 20 \\
& 12 & $164 / 243$ & 20 \\
$($ for $n \geq 6)$ & $3 n-3$ & $109 / 162$ & 20 \\
$($ for $n \geq 6)$ & $3 n-2$ & $163 / 243$ & 20 \\
(for $n \geq 6)$ & $3 n-1$ & $2 / 3+2 / 3^{n}$ & $3 n+2$ \\
& 14 & $\left.2 / 3 \cdot 3^{n-1}\right)$ & $3 n+2$ \\
& \multicolumn{3}{|c}{} \\
\hline
\end{tabular}

down the set of $n$ with $\|n\|=k$ corresponds to going up the set of defects $\delta(n)$ of $n$ with $\|n\|=k$; and assuming $k$ is large enough relative to how far up or down you want to go, this is just looking at $\mathscr{D}^{k}$. And if we count up one at a time, then - again, assuming $k$ is sufficiently large relative to how far out we count - we will stay within the initial $\omega$ of $\mathscr{D}^{k}$. So with a classification of numbers $n$ such that $D(n) \leq 1$, one can determine the $E_{r}(k)$. (Indeed, one can also do the reverse.)

Note that Theorem 1.10 also works for $k=6$, so if one wants to break it down by the residue of $k$ modulo 3 , one could say it works for $k \geq 6$ with $k \equiv 0(\bmod 3)$, for $k \geq 8$ with $k \equiv 2(\bmod 3)$, and for $k \geq 10$ with $k \equiv 1(\bmod 3)$. (Indeed, this is 
TABLE 3 . Table of $h_{r}$ and $K_{r}$ for $k \equiv 1(\bmod 3)$ with $k>1$.

\begin{tabular}{|c|c|c|c|}
\hline & $r$ & $\overline{h_{r, 1}}$ & $K_{r, 1}$ \\
\hline & 0 & 1 & 4 \\
\hline & 1 & $8 / 9$ & 10 \\
\hline & 2 & $5 / 6$ & 10 \\
\hline & 3 & $64 / 81$ & 16 \\
\hline & 4 & $7 / 9$ & 16 \\
\hline & 5 & $41 / 54$ & 16 \\
\hline (for $n \geq 4)$ & $n+2$ & $3 / 4+1 /\left(4 \cdot 3^{n}\right)$ & $3 n+4$ \\
\hline
\end{tabular}

what we have done in Tables 1, 2, and 3,) Note how all three of these correspond to $k$ exactly large enough for $E(k)$ to be divisible by 9 , as per the last part of Theorem 1.12

One thing worth noting here is that the formulae for $E_{0}(k)$ and $E_{1}(k)$, as originally proven by Selfridge and Rawsthorne respectively, were both originally proven directly by induction on $k$. Whereas here we have proven Theorem 1.12 by a different method, namely, analysis of defects. (Although this analysis of defects in turn depends on Rawsthorne's formula for $E_{1}(k)$ to serve as a base case; see [6].) This raises the question of whether a similar inductive proof for general $E_{r}(k)$ could be done now that the formulae for them are known. (In fact this author originally proved these formulae by a different method entirely, that of analyzing certain transformations of expression, so other methods certainly are possible.)

1.3. Low-defect polynomials and numbers of low defect. In order to prove Theorem 1.8, we make use of the idea of low-defect polynomials from [3, 4. A lowdefect polynomial is a particular type of multilinear polynomial; see Section 2 for details. In $[3]$ it is proved that, given any positive real number $s$, one can write down a finite set of low-defect polynomials $\mathcal{S}$ such that every number $n$ with $\delta(n)<s$ can be written in the form $f\left(3^{n_{1}}, \ldots, 3^{n_{d}}\right) 3^{n_{d+1}}$ for some $f \in \mathcal{S}$; and that, moreover, such an $n$ can always be represented "efficiently" in such a fashion. Moreover, one can choose $\mathcal{S}$ such that for any $f \in \mathcal{S}$, one has $\operatorname{deg} f \leq s$. (Note that the degree of a low-defect polynomial is always equal to the number of variables it is in, since low-defect polynomials are multilinear and always include a term containing all the variables.)

Using this fact about low-defect polynomials, this author proved in [3] that the set $\mathscr{D}$ is well-ordered with order type $\omega^{\omega}$, as well as the more specific Theorem 1.7 mentioned above, and other results mentioned above such as that the limit of the initial $\omega^{k}$ defects is equal to $k$. However, this is not enough to prove the more specific theorems shown in this paper, such as Theorem [1.9, But in [4] an improvement was shown, that we can in fact take $\mathcal{S}$ such that for all $f \in \mathscr{T}$, one has $\delta(f) \leq s$; here $\delta(f)$ is a number that bounds above $\delta(n)$ for any $n$ represented by $f$ in the fashion described above; again, see 2 for more on this.

On top of that, it was shown in [4 that $\delta(f) \geq \operatorname{deg} f+\delta(m)$, where $m$ is the leading coefficient of $f$. Putting this together, one gets the inequality

$$
\operatorname{deg} f+\delta(m) \leq s .
$$

It's this stronger inequality that allows us to prove Theorem 1.8, where the inequality $\operatorname{deg} f \leq s$ would not be enough. To see why this inequality is so helpful, 
say we're given $s$ and we pick $\mathcal{S}$ as described above. Then if $f \in \mathcal{S}$, one of two things must be true: Either $\operatorname{deg} f<\lfloor s\rfloor$, in which case $f$ does not make much of a contribution to $\mathscr{D} \cap[0, s)$ compared to polynomials of higher degree; or $\operatorname{deg} f=\lfloor s\rfloor$, in which case $\delta(m)$ is at most the fractional part of $r$, a number which is less than 1. Since there are only finitely many defects below any given number less than 1 , this puts substantial constraints on $m$ and therefore on $f$, in ways that the weaker inequality $\operatorname{deg} f \leq s$ does not. This allows us to prove Theorem 1.9 .

Note that the method we use to turn the results of [4 into Theorem 1.8 actually has much more power than we use in this paper; but an exploration of the full power of this method would take us too far away from the subject of $D(n)$, and so will be detailed in a future paper [5].

1.4. A quick note on stabilization. An important property satisfied by integer integer complexity is the phenomenon of stabilization. Because one has $\left\|3^{k}\right\|=3 k$ for $k>1$, as well as that $\left\|2 \cdot 3^{k}\right\|=2+3 k$ and $\left\|4 \cdot 3^{k}\right\|=4+3 k$, one might hope that in general the equation $\|3 n\|=\|n\|+3$ holds for all $n>1$. Unfortunately that is not the case; for instance, for $n=107$, one has $\|107\|=16$, but $\|321\|=18$. Another counterexample is $n=683$, for which one has $\|683\|=22$, but $\|2049\|=23$. There are even cases where $\|3 n\|<\|n\|$, such as $n=4721323$, which has $\|3 n\|=\|n\|-1$.

And yet the initial hope is not entirely in vain. In [6], it was proved:

Theorem 1.13. For any natural number $n$, there exists $K \geq 0$ such that, for any $k \geq K$

$$
\left\|3^{k} n\right\|=3(k-K)+\left\|3^{K} n\right\| .
$$

Based on this, we define:

Definition 1.14. A number $m$ is called stable if $\left\|3^{k} m\right\|=3 k+\|m\|$ holds for every $k \geq 0$. Otherwise it is called unstable.

So, we can restate Theorem 1.13 by saying, for any $n$, there is some $K$ such that $3^{K} n$ is stable.

This allows us to define stable or stabilized analogues of many of the concepts and discussed above, and prove stabilized analogues of the theorems discussed in Section 1.1. See Sections 2.1 and 3 for the relevant definitions, and Section 4 for the versions of the main theorems generalized to cover the stabilized case as well.

1.5. Discussion: Comparison to addition chains. In order to make sense of Theorem 1.8, it is helpful to introduce an analogy to addition chains, a different notion of complexity which is similar in spirit but different in detail. An addition chain for $n$ is defined to be a sequence $\left(a_{0}, a_{1}, \ldots, a_{r}\right)$ such that $a_{0}=1, a_{r}=n$, and, for any $1 \leq k \leq r$, there exist $0 \leq i, j<k$ such that $a_{k}=a_{i}+a_{j}$; the number $r$ is called the length of the addition chain. The shortest length among addition chains for $n$, called the addition chain length of $n$, is denoted $\ell(n)$. Addition chains were introduced in 1894 by H. Dellac [12 and reintroduced in 1937 by A. Scholz [19]; extensive surveys on the topic can be found in Knuth [16, Section 4.6.3] and Subbarao [20].

The notion of addition chain length has obvious similarities to that of integer complexity; each is a measure of the resources required to build up the number $n$ starting from 1 . Both allow the use of addition, but integer complexity supplements this by allowing the use of multiplication, while addition chain length supplements 
this by allowing the reuse of any number at no additional cost once it has been constructed. Furthermore, both measures are approximately logarithmic; the function $\ell(n)$ satisfies

$$
\log _{2} n \leq \ell(n) \leq 2 \log _{2} n .
$$

A difference worth noting is that $\ell(n)$ is actually known to be asymptotic to $\log _{2} n$, as was proved by Brauer [8], but the function $\|n\|$ is not known to be asymptotic to $3 \log _{3} n$; the value of the quantity $\lim \sup _{n \rightarrow \infty} \frac{\|n\|}{\log n}$ remains unknown.

Nevertheless, there are important similarities between integer complexity and addition chains. As mentioned above, the set of all integer complexity defects is a well-ordered subset of the real numbers, with order type $\omega^{\omega}$. We might also define the notion of addition chain defect, defined by

$$
\delta^{\ell}(n):=\ell(n)-\log _{2} n ;
$$

for as shown in [1, the well-ordering theorem for integer complexity has an analogue for addition chains:

Theorem 1.15 (Addition chain well-ordering theorem). Let $\mathscr{D}^{\ell}$ denote the set $\left\{\delta^{\ell}(n): n \in \mathbb{N}\right\}$. Then considered as a subset of the real numbers, $\mathscr{D}^{\ell}$ is wellordered and has order type $\omega^{\omega}$.

More commonly, however, it is not $\delta^{\ell}(n)$ that has been studied, but rather $s(n)$, the number of small steps of $n$, which is defined to be $\ell(n)-\left\lfloor\log _{2}\right\rfloor$, or equivalently $\left\lceil\delta^{\ell}(n)\right\rceil$. The quantity $D(n)$ that we introduce seems to play a role in integer complexity similar to $s(n)$ in the study of addition chains. Now, unlike with $s(n)$ and $\delta^{\ell}(n), D(n)$ is not simply $\lceil\delta(n)\rceil$; for instance, $D(56)=1$ even though $\delta(56)>$ 1. (Although Theorem 3.12 will show how $D(n)$ is in a certain sense almost $\lceil\delta(n)\rceil$.) But, there are further analogies.

Analogous to Theorem 1.13, we have (from [1]) the following:

Theorem 1.16. For any natural number $n$, there exists $K \geq 0$ such that, for any $k \geq K$,

$$
\ell\left(2^{k} n\right)=(k-K)+\ell\left(2^{K} n\right) .
$$

So we define a number $n$ to be $\ell$-stable if for any $k$, one has $\ell\left(2^{k} n\right)=k+\ell(n)$; then Theorem 1.16 says that for any $n$, there is some $K$ such that $2^{K} n$ is $\ell$-stable. This allows us to formulate a stabilized version of the previous analogy - and of the ones to follow.

In [1, this author conjectured:

Conjecture 1.17. For each whole number $k, \mathscr{D}^{\ell} \cap[0, k]$ has order type $\omega^{k}$.

In other words, this conjecture states that the limit of the initial $\omega^{k}$ addition chain defects is equal to $k$. If true, this would mean that $s(n)$ plays the same role for $\mathscr{D}^{\ell}$ as $D(n)$ does for the $\mathscr{D}^{a}$, that $s(n)$ is the smallest $k$ such that the order type of $\mathscr{D}^{\ell} \cap\left[0, \delta^{\ell}(n)\right)$ is less than $\omega^{k}$.

One similarly based on conjectures in [1] gets analogies between $D_{s t}(n)$ and $s_{s t}(n)$ and how they determine position in $\mathscr{D}_{s t}^{a}$ and $\mathscr{D}_{s t}^{\ell}$, respectively; see Section 3 for definitions of these.

It's worth noting here one important difference between these two cases: in the integer complexity case, we need to split things into congruence classes modulo 3 based on $\|n\|$. This has no analogue in the addition chain case. The difference comes from a difference in certain fundamental inequalities that these quantities obey. 
Integer complexity obeys $\|3 n\| \leq\|n\|+3$, with equality if and only if $\delta(3 n)=\delta(n)$. The addition chain analogue of this is that one has $\ell(2 n) \leq \ell(n)+1$, with equality if and only if $\delta^{\ell}(2 n)=\delta^{\ell}(n)$. The result [1, 6] is that if we have two numbers $m$ and $n$ with $\delta^{\ell}(n)=\delta^{\ell}(m)$, then one must have $m=2^{k} n$ for some $k \in \mathbb{Z}$; and if we have two numbers $m$ and $n$ with $\delta(n)=\delta(m)$, then one must have $m=3^{k} n$ for some $k \in \mathbb{Z}$. However in the latter case we must also have $\|m\| \equiv\|n\|(\bmod 3)$; this is why the sets $\mathscr{D}^{a}$ are disjoint. In the addition chain case there is no such congruence requirement; $\ell(n)$ and $\ell(m)$ need only be congruent modulo 1 , which is no requirement at all, so splitting up $\mathscr{D}^{\ell}$ in a similar manner does not make sense. The set $\mathscr{D}^{\ell}$ already covers the one and only congruence class that exists in the addition chain case.

But it is not only our primary theorem but also our secondary theorem here that has an analogues for addition chains, and in this case the analogy does not rely on any conjectures. While the hypothesis that the order type of $\mathscr{D}^{\ell} \cap[0, k]$ is equal to $\omega^{k}$ remains a conjecture, that this holds for $k \leq 2-$ and in particular that it holds for $k=1$ - was proven in [1. This means that just as we can look at the first $\omega$ elements of each $\mathscr{D}^{a}$ in order to determine the $r^{\prime}$ th-highest number of complexity $k$, we can look at the first $\omega$ elements of $\mathscr{D}^{\ell}$ to determine the $r$ 'th-highest number of addition chain length $k$ (or at most $k$, which in these cases is the same thing). (Again, here $k$ must be sufficiently large relative to $r$. Also, again here we are using the convention that $r$ starts at 0 rather than 1.)

Specifically, it's an easy corollary of the classification of numbers with $s(n) \leq 1$ (due to Gioia et al. [13) that:

Theorem 1.18. For $k \geq r+1$ (or for $k \geq 0$ when $r=0$ ), the $r$ 'th-largest number of addition chain length $k$ is $\left(\frac{1}{2}+\frac{1}{2^{r+1}}\right) 2^{k}$.

Obviously here the fraction $\frac{1}{2}+\frac{1}{2^{r+1}}$ plays the role of the $h_{r}$ and $r+1$ plays the role of $K_{r}$; unlike with integer complexity, there are no irregularities here, just a single straightforward infinite family. (And note how the analogue of the $K_{r}$ increases in what is mostly steps of 1 , rather than mostly steps of 3 like the actual $K_{r}$, because once again with addition chains there's only one congruence class.) For more on the analogy between integer complexity and addition chains, particularly with regard to their sets of defects, one may see [4].

\section{INTEGER COMPLEXITY, WELL-ORDERING, AND LOW-DEFECT POLYNOMIALS}

In this section we summarize the results of [3, 4, 6] that we will need later regarding the defect $\delta(n)$; the stable complexity $\|n\|_{s t}$ and stable defect $\delta_{s t}(n)$ described below; and low-defect polynomials.

2.1. The defect and stability. First, some basic facts about the defect:

Theorem 2.1. We have:

(1) For all $n, \delta(n) \geq 0$.

(2) For $k \geq 0, \delta\left(3^{k} n\right) \leq \delta(n)$, with equality if and only if $\left\|3^{k} n\right\|=3 k+\|n\|$. The difference $\delta(n)-\delta\left(3^{k} n\right)$ is a nonnegative integer.

(3) A number $n$ is stable if and only if for any $k \geq 0, \delta\left(3^{k} n\right)=\delta(n)$.

(4) If the difference $\delta(n)-\delta(m)$ is rational, then $n=m 3^{k}$ for some integer $k$ (and so $\delta(n)-\delta(m) \in \mathbb{Z}$ ).

(5) Given any $n$, there exists $k$ such that $3^{k} n$ is stable. 
(6) For a given defect $\alpha$, the set $\{m: \delta(m)=\alpha\}$ has either the form $\left\{n 3^{k}\right.$ : $0 \leq k \leq L\}$ for some $n$ and $L$, or the form $\left\{n 3^{k}: 0 \leq k\right\}$ for some $n$. The latter occurs if and only if $\alpha$ is the smallest defect among $\delta\left(3^{k} n\right)$ for $k \in \mathbb{Z}$.

(7) If $\delta(n)=\delta(m)$, then $\|n\|=\|m\|(\bmod 3)$.

(8) $\delta(1)=1$, and for $k \geq 1, \delta\left(3^{k}\right)=0$. No other integers occur as $\delta(n)$ for any $n$.

(9) If $\delta(n)=\delta(m)$ and $n$ is stable, then so is $m$.

Proof. Parts (1) through (8), excepting part (3), are just Theorem 2.1 from 3 . Part (3) is Proposition 12 from [6], and part (9) is Proposition 3.1 from [3].

We will want to consider the set of all defects:

Definition 2.2. We define the defect set $\mathscr{D}$ to be $\{\delta(n): n \in \mathbb{N}\}$, the set of all defects.

We also defined $\mathscr{D}^{a}$, for $a$ a congruence class modulo 3 , in Definition 1.5 earlier.

The paper [3] also defined the notion of a stable defect:

Definition 2.3. We define a stable defect to be the defect of a stable number, and define $\mathscr{D}_{s t}$ to be the set of all stable defects. Also, for $a$ a congruence class modulo 3, we define $\mathscr{D}_{s t}^{a}=\mathscr{D}^{a} \cap \mathscr{D}_{s t}$.

Because of part (9) of Theorem 2.1, this definition makes sense; a stable defect $\alpha$ is not just one that is the defect of some stable number, but one for which any $n$ with $\delta(n)=\alpha$ is stable. Stable defects can also be characterized by the following proposition from [3]:

Proposition 2.4. A defect $\alpha$ is stable if and only if it is the smallest $\beta \in \mathscr{D}$ such that $\beta \equiv \alpha(\bmod 1)$.

We can also define the stable defect of a given number, which we denote $\delta_{s t}(n)$.

Definition 2.5. For a positive integer $n$, define the stable defect of $n$, denoted $\delta_{s t}(n)$, to be $\delta\left(3^{k} n\right)$ for any $k$ such that $3^{k} n$ is stable. (This is well-defined as if $3^{k} n$ and $3^{\ell} n$ are stable, then $k \geq \ell$ implies $\delta\left(3^{k} n\right)=\delta\left(3^{\ell} n\right)$, and $\ell \geq k$ implies this as well.)

Note that the statement " $\alpha$ is a stable defect", which earlier we were thinking of as " $\alpha=\delta(n)$ for some stable $n$ ", can also be read as the equivalent statement " $\alpha=\delta_{s t}(n)$ for some $n$ ".

Similarly we have the stable complexity:

Definition 2.6. For a positive integer $n$, define the stable complexity of $n$, denoted $\|n\|_{s t}$, to be $\left\|3^{k} n\right\|-3 k$ for any $k$ such that $3^{k} n$ is stable.

We then have the following facts relating the notions of $\|n\|, \delta(n),\|n\|_{s t}$, and $\delta_{s t}(n)$ :

Proposition 2.7. We have:

(1) $\delta_{s t}(n)=\min _{k \geq 0} \delta\left(3^{k} n\right)$

(2) $\delta_{s t}(n)$ is the smallest $\alpha \in \mathscr{D}$ such that $\alpha \equiv \delta(n)(\bmod 1)$.

(3) $\|n\|_{s t}=\min _{k \geq 0}\left(\left\|3^{k} n\right\|-3 k\right)$

(4) $\delta_{s t}(n)=\|n\|_{s t}-3 \log _{3} n$

(5) $\delta_{s t}(n) \leq \delta(n)$, with equality if and only if $n$ is stable. 
(6) $\|n\|_{s t} \leq\|n\|$, with equality if and only if $n$ is stable.

(7) $\|3 n\|_{s t}=\|n\|_{s t}+3$

(8) If $\delta_{s t}(n)=\delta_{s t}(m)$, then $\|n\|_{s t} \equiv\|m\|_{s t}(\bmod 3)$.

Proof. Statements (1)-(6) are just Propositions 3.5, 3.7, and 3.8 from [3]. Statement (7) follows from the definition of stable complexity; if $3^{k} n$ is stable, then $\|3 n\|_{s t}=$ $\left\|3^{k} n\right\|-3(k-1)=\left\|3^{k} n\right\|-3 k+3=\|n\|_{s t}+3$. To prove statement (8), note that if $\delta_{s t}(n)=\delta_{s t}(m)$, then by statement $(2)$ one has $\delta(n) \equiv \delta(m)(\bmod 1)$, and so by Propostion [2.1. one has that $n=m 3^{k}$ for some $k \in \mathbb{Z}$, and so $\|n\|_{s t}=$ $\|m\|_{s t}+3 k$.

Note, by the way, that just as $\mathscr{D}_{s t}$ can be characterized either as defects $\delta(n)$ with $n$ stable or as defects $\delta_{s t}(n)$ for any $n, \mathscr{D}_{s t}^{a}$ can be characterized either as defects $\delta(n)$ with $n$ stable and $\|n\| \equiv a(\bmod 3)$, or as defects $\delta_{s t}(n)$ for any $n$ with $\|n\|_{s t} \equiv a(\bmod 3)$.

Three defects that will be particularly important in this paper are the smallest three defects:

\section{Proposition 2.8 .}

$$
\mathscr{D} \cap[0,2 \delta(2)]=\{0, \delta(2), 2 \delta(2)\} .
$$

Proof. Proposition 37 from 6 tells us that the only leaders with defect less than $3 \delta(2)$ are 3,2 , and 4 , which respectively have defects $0, \delta(2)$, and $2 \delta(2)$.

2.2. Low-defect polynomials. As has been mentioned in Section 1.3, we are going to represent the set of numbers with defect at most $r$ by substituting in powers of 3 into certain multilinear polynomials we call low-defect polynomials. We will associate with each one a "base complexity" to form a low-defect pair. In this section we will review the basic properties of these polynomials. First, their definition:

Definition 2.9. We define the set $\mathscr{P}$ of low-defect pairs as the smallest subset of $\mathbb{Z}\left[x_{1}, x_{2}, \ldots\right] \times \mathbb{N}$ such that:

(1) For any constant polynomial $k \in \mathbb{N} \subseteq \mathbb{Z}\left[x_{1}, x_{2}, \ldots\right]$ and any $C \geq\|k\|$, we have $(k, C) \in \mathscr{P}$.

(2) Given $\left(f_{1}, C_{1}\right)$ and $\left(f_{2}, C_{2}\right)$ in $\mathscr{P}$, we have $\left(f_{1} \otimes f_{2}, C_{1}+C_{2}\right) \in \mathscr{P}$, where, if $f_{1}$ is in $d_{1}$ variables and $f_{2}$ is in $d_{2}$ variables,

$$
\left(f_{1} \otimes f_{2}\right)\left(x_{1}, \ldots, x_{d_{1}+d_{2}}\right):=f_{1}\left(x_{1}, \ldots, x_{d_{1}}\right) f_{2}\left(x_{d_{1}+1}, \ldots, x_{d_{1}+d_{2}}\right) .
$$

(3) Given $(f, C) \in \mathscr{P}, c \in \mathbb{N}$, and $D \geq\|c\|$, we have $\left(f \otimes x_{1}+c, C+D\right) \in \mathscr{P}$ where $\otimes$ is as above.

The polynomials obtained this way will be referred to as low-defect polynomials. If $(f, C)$ is a low-defect pair, $C$ will be called its base complexity. If $f$ is a lowdefect polynomial, we will define its absolute base complexity, denoted $\|f\|$, to be the smallest $C$ such that $(f, C)$ is a low-defect pair. We will also associate to a low-defect polynomial $f$ the augmented low-defect polynomial

$$
\hat{f}=f \otimes x_{1} ;
$$

if $f$ is in $d$ variables, this is $f x_{d+1}$. 
In this paper we will only concern ourselves with low-defect pairs $(f, C)$ where $C=\|f\|$, so in the remainder of what follows, we will mostly dispense with the formalism of low-defect pairs and just discuss low-defect polynomials.

Note that the degree of a low-defect polynomial is also equal to the number of variables it uses; see Proposition 2.10. Also note that augmented low-defect polynomials are never themselves low-defect polynomials; as we will see in a moment (Proposition 2.10), low-defect polynomials always have nonzero constant term, whereas augmented low-defect polynomials always have zero constant term. We can also observe that low-defect polynomials are in fact read-once polynomials as discussed in for instance [21].

Note that we do not really care about what variables a low-defect polynomial is in - if we permute the variables of a low-defect polynomial or replace them with others, we will still regard the result as a low-defect polynomial. From this perspective, the meaning of $f \otimes g$ could be simply regarded as "relabel the variables of $f$ and $g$ so that they do not share any, then multiply $f$ and $g$ ". Helpfully, the $\otimes$ operator is associative not only with this more abstract way of thinking about it, but also in the concrete way it was defined above.

In [3] were proved the following propositions about low-defect polynomials:

Proposition 2.10. Suppose $f$ is a low-defect polynomial of degree $d$. Then $f$ is a polynomial in the variables $x_{1}, \ldots, x_{d}$, and it is a multilinear polynomial, i.e., it has degree 1 in each of its variables. The coefficients are non-negative integers. The constant term is nonzero, and so is the coefficient of $x_{1} \cdots x_{d}$, which we will call the leading coefficient of $f$.

Proof. This is Proposition 4.2 from $[3]$.

Proposition 2.11. If $f$ is a low-defect polynomial of degree $d$, then

$$
\left\|f\left(3^{n_{1}}, \ldots, 3^{n_{d}}\right)\right\| \leq\|f\|+3\left(n_{1}+\ldots+n_{d}\right) .
$$

and

$$
\left\|\hat{f}\left(3^{n_{1}}, \ldots, 3^{n_{d+1}}\right)\right\| \leq\|f\|+3\left(n_{1}+\ldots+n_{d+1}\right) .
$$

Proof. This is a combination of Proposition 4.5 and Corollary 4.12 from 3 .

The above proposition motivates the following definition:

Definition 2.12. Given a low-defect polynomial $f$ (say of degree $d$ ) and a number $N$, we will say that $f$ efficiently 3 -represents $N$ if there exist nonnegative integers $n_{1}, \ldots, n_{d}$ such that

$$
N=f\left(3^{n_{1}}, \ldots, 3^{n_{d}}\right) \text { and }\|N\|=\|f\|+3\left(n_{1}+\ldots+n_{d}\right) .
$$

We will say $\hat{f}$ efficiently 3 -represents $N$ if there exist $n_{1}, \ldots, n_{d+1}$ such that

$$
N=\hat{f}\left(3^{n_{1}}, \ldots, 3^{n_{d+1}}\right) \text { and }\|N\|=\|f\|+3\left(n_{1}+\ldots+n_{d+1}\right) .
$$

More generally, we will also say $f 3$-represents $N$ if there exist nonnegative integers $n_{1}, \ldots, n_{d}$ such that $N=f\left(3^{n_{1}}, \ldots, 3^{n_{d}}\right)$. and similarly with $\hat{f}$.

Note that previous papers [2, 3, 4] instead spoke of a low-defect pair $(f, C)$ efficiently 3 -representing a number $N$; however, as mentioned in those papers, it is only possible for some $(f, C)$ to efficiently 3-represent a number $N$ if in fact $C=\|f\|$, so there is no loss here. 
In keeping with the name, numbers 3-represented by low-defect polynomials, or their augmented versions, have bounded defect. Let us make some definitions first:

Definition 2.13. Given a low-defect polynomial $f$ we define $\delta(f)$, the defect of $f$, to be $\|f\|-3 \log _{3} m$, where $m$ is the leading coefficient of $f$.

Definition 2.14. Given a low-defect polynomial $f$ of degree $d$, we define

$$
\delta_{f}\left(n_{1}, \ldots, n_{d}\right)=\|f\|+3\left(n_{1}+\ldots+n_{d}\right)-3 \log _{3} f\left(3^{n_{1}}, \ldots, 3^{n_{d}}\right) .
$$

Then we have:

Proposition 2.15. Let $f$ be a low-defect polynomial of degree $d$, and let the numbers $n_{1}, \ldots, n_{d+1}$ be nonnegative integers.

(1) We have

$$
\delta\left(\hat{f}\left(3^{n_{1}}, \ldots, 3^{n_{d+1}}\right)\right) \leq \delta_{f}\left(n_{1}, \ldots, n_{d}\right),
$$

and the difference is an integer.

(2) We have

$$
\delta_{f}\left(n_{1}, \ldots, n_{d}\right) \leq \delta(f),
$$

and if $d \geq 1$, this inequality is strict.

(3) The function $\delta_{f}$ is strictly increasing in each variable, and

$$
\delta(f)=\sup _{n_{1}, \ldots, n_{d}} \delta_{f}\left(n_{1}, \ldots, n_{d}\right) .
$$

Proof. This is a combination of Proposition 4.9 and Corollary 4.14 from [3] and Proposition 2.15 from [4].

Importantly, the set of defects coming from a low-defect polynomial of degree $r$ has order type approximately $\omega^{r}$; if rather than the actual defects we use $\delta_{f}$, then this is exact. More formally:

Proposition 2.16. Let $f$ be a low-defect polynomial of degree $d$. Then:

(1) The image of $\delta_{f}$ is a well-ordered subset of $\mathbb{R}$, with order type $\omega^{d}$.

(2) The set of $\delta(N)$ for all $N$ 3-represented by the augmented low-defect polynomial $\hat{f}$ is a well-ordered subset of $\mathbb{R}$, with order type at least $\omega^{d}$ and at most $\omega^{d}(\lfloor\delta(f)\rfloor+1)<\omega^{d+1}$. The same is true if $f$ is used instead of the augmented version $\hat{f}$.

Proof. This is a combination of Propositions 6.2 and 6.3 from 3 .

The second part of the above proposition follows from the first by means of theorems about cutting and pasting of well-ordered sets, ultimately due to Carruth 9. In particular:

Proposition 2.17. We have:

(1) If $S$ is a well-ordered set and $S=S_{1} \cup \ldots \cup S_{n}$, and $S_{1}$ through $S_{n}$ all have order type less than $\omega^{k}$, then so does $S$.

(2) If $S$ is a well-ordered set of order type $\omega^{k}$ and $S=S_{1} \cup \ldots \cup S_{n}$, then at least one of $S_{1}$ through $S_{n}$ also has order type $\omega^{k}$.

Proof. One may see [9] or [11] for proofs of these.

We will need in particular the following variant: 
Proposition 2.18. Suppose $\alpha$ is an ordinal and $S$ is a well-ordered set which can be written as a finite union $S_{1} \cup \ldots \cup S_{k}$ such that:

(1) The $S_{i}$ all have order types at most $\omega^{\alpha}$.

(2) If a set $S_{i}$ has order type $\omega^{\alpha}$, it is cofinal in $S$.

Then the order type of $S$ is at most $\omega^{\alpha}$. In particular, if at least one of the $S_{i}$ has order type $\omega^{\alpha}$, then $S$ has order type $\omega^{\alpha}$.

Proof. A proof of this can be found in [1] where it is Proposition 5.4.

As was noted above, we have $\delta\left(f\left(3^{n_{1}}, \ldots, 3^{n_{d}}\right) \leq \delta_{f}\left(n_{1}, \ldots, n_{d}\right)\right.$. Importantly, though, for certain low-defect polynomials $f$, namely, those with $\delta(f)<\operatorname{deg} f+1$, we can show that equality holds for "most" choices of $\left(n_{1}, \ldots, n_{d}\right)$ in a certain sense.

Specifically:

Proposition 2.19. Let $f$ be a low-defect polynomial of degree $d$ with $\delta(f)<d+1$. Define its "exceptional set" to be

$$
S:=\left\{\left(n_{1}, \ldots, n_{d}\right):\left\|f\left(3^{n_{1}}, \ldots, 3^{n_{d}}\right)\right\|_{s t}<\|f\|+3\left(n_{1}+\ldots+n_{d}\right)\right\}
$$

Then the set $\left\{\delta\left(f\left(3^{n_{1}}, \ldots, 3^{n_{d}}\right)\right):\left(n_{1}, \ldots, n_{d}\right) \in S\right\}$ has order type less than $\omega^{d}$, and therefore so does the set $\left\{\delta\left(\hat{f}\left(3^{n_{1}}, \ldots, 3^{n_{d+1}}\right)\right):\left(n_{1}, \ldots, n_{d}\right) \in S\right\}$. In particular, for $a \not \equiv\|f\|(\bmod 3)$, the set

$$
\left\{\delta\left(\hat{f}\left(3^{n_{1}}, \ldots, 3^{n_{d+1}}\right)\right):\left(n_{1}, \ldots, n_{d+1}\right) \in \mathbb{Z}_{\geq 0}^{d+1}\right\} \cap \mathscr{D}^{a}
$$

has order type less than $\omega^{d}$. Meanwhile, the set

$$
\left\{\delta\left(f\left(3^{n_{1}}, \ldots, 3^{n_{d}}\right)\right):\left(n_{1}, \ldots, n_{d}\right) \notin S\right\}
$$

has order type at least $\omega^{d}$, and thus so does the set

$$
\left\{\delta\left(f\left(3^{n_{1}}, \ldots, 3^{n_{d}}\right)\right):\left(n_{1}, \ldots, n_{d}\right) \in \mathbb{Z}_{\geq 0}^{d}\right\} \cap \mathscr{D}_{\text {st }}^{\|f\|} ;
$$

moreover, the supremum of this latter set is equal to $\delta(f)$.

Proof. Most of this is direct from Proposition 7.2 from [3] the only parts not covered in the statement there there are the statement about $\left\{\delta\left(\hat{f}\left(3^{n_{1}}, \ldots, 3^{n_{d+1}}\right)\right)\right.$ : $\left.\left(n_{1}, \ldots, n_{d}\right) \in S\right\}$, the statement regarding $a \not \equiv\|f\|(\bmod 3)$, and the final statement.

The first of these follows directly from the first part, because

$$
\delta\left(\hat{f}\left(3^{n_{1}}, \ldots, 3^{n_{d+1}}\right)\right) \leq \delta\left(f\left(3^{n_{1}}, \ldots, 3^{n_{d}}\right)\right)
$$

with the difference being an integer, and that integer can certainly be no more than $\delta\left(f\left(3^{n_{1}}, \ldots, 3^{n_{d}}\right)\right) \leq \delta(f)$. Thus the set

$$
\left\{\delta\left(\hat{f}\left(3^{n_{1}}, \ldots, 3^{n_{d+1}}\right)\right):\left(n_{1}, \ldots, n_{d+1}\right) \in \mathbb{Z}_{\geq 0}^{d+1}\right\} \cap \mathscr{D}^{a}
$$

can be covered by finitely many translates of $\left\{\delta\left(f\left(3^{n_{1}}, \ldots, 3^{n_{d}}\right)\right):\left(n_{1}, \ldots, n_{d}\right) \in S\right\}$ and so by Proposition 2.17 has order type less than $\omega^{d}$.

For the statement about

$$
\left\{\delta\left(\hat{f}\left(3^{n_{1}}, \ldots, 3^{n_{d+1}}\right)\right):\left(n_{1}, \ldots, n_{d+1}\right) \in \mathbb{Z}_{\geq 0}^{d+1}\right\} \cap \mathscr{D}^{a}
$$

with $a \not \equiv C(\bmod 3)$, if $\left\|\hat{f}\left(3^{n_{1}}, \ldots, 3^{n_{d}}\right)\right\| \equiv a \not \equiv\|f\|(\bmod 3)$, then in particular this means that

$$
\left\|\hat{f}\left(3^{n_{1}}, \ldots, 3^{n_{d+1}}\right)\right\| \neq\|f\|+3\left(n_{1}+\ldots+n_{d+1}\right)
$$


which means that

$$
\left\|\hat{f}\left(3^{n_{1}}, \ldots, 3^{n_{d+1}}\right)\right\|<\|f\|+3\left(n_{1}+\ldots+n_{d+1}\right)
$$

and therefore that

$$
\left\|f\left(3^{n_{1}}, \ldots, 3^{n_{d}}\right)\right\|_{s t}<\|f\|+3\left(n_{1}+\ldots+n_{d}\right)
$$

i.e., that $\left(n_{1}, \ldots, n_{d}\right) \in S$. Applying what was proved in the previous paragraph now proves the statement.

As for the final statement, the set $\left\{\delta\left(f\left(3^{n_{1}}, \ldots, 3^{n_{d}}\right)\right):\left(n_{1}, \ldots, n_{d}\right) \in \mathbb{Z}_{\geq 0}^{d}\right\} \cap$ $\mathscr{D}_{s t}^{\|f\|}$ contains $\delta_{f}\left(\mathbb{N}^{d} \backslash S\right.$ ) (one may see the proof in [3]) which in turn contains $\delta_{f}\left(\mathbb{N}^{d}\right) \backslash \delta_{f}(S)$. Since the image of $\delta_{f}$ has order type $\omega^{d}$ while $\delta_{f}(S)$ has order type less than $\omega^{d}$ - similarly to above, this follows by the initial statement and Proposition 2.17 - it follows that $\delta_{f}\left(\mathbb{N}^{d}\right) \backslash \delta_{f}(S)$ has order type $\omega^{d}$ and thus is cofinal in the image of $\delta_{f}$, and thus has supremum $\delta(f)$; and the same is true of the larger set $\left\{\delta\left(f\left(3^{n_{1}}, \ldots, 3^{n_{d}}\right)\right):\left(n_{1}, \ldots, n_{d}\right) \in \mathbb{Z}_{\geq 0}^{d}\right\} \cap \mathscr{D}_{s t}^{\|f\|}$ which is also bounded above by $\delta(f)$.

Finally, one more property of low-defect polynomials we will need is the following:

Proposition 2.20. Let $f$ be a low-defect polynomial, and suppose that $a$ is the leading coefficient of $f$. Then $\|f\| \geq\|a\|+\operatorname{deg} f$. In particular, $\delta(f) \geq \delta(a)+\operatorname{deg} f$.

Proof. This is Proposition 3.24 from [4].

With this, we have the basic properties of low-defect polynomials.

Remark 2.21. Note that one reason nothing is lost here by discarding the formalism of low-defect pairs is that the low-defect pairs $(f, C)$ we will (implicitly) concern ourselves with in this paper are ones that satisfy $C-3 \log _{3} m<\operatorname{deg} f+1$, where $m$ is the leading coefficient of $f$. However, by Proposition 2.20 .

$$
\operatorname{deg} f \leq \delta(f) \leq C-3 \log _{3} m<\operatorname{deg} f+1,
$$

thus $C-\|f\|=\left(C-3 \log _{3} m\right)-\delta(f)<1$ and so $C=\|f\|$. So if we were to use low-defect pairs, we would only be using pairs where $C=\|f\|$, so we lose nothing by making this assumption.

2.3. Good coverings. We need one more set of definitions before we can state the theorem that will be used as the basis of the proof of the main theorem. We define:

Definition 2.22. A natural number $n$ is called a leader if it is the smallest number with a given defect. By part (6) of Theorem 2.1, this is equivalent to saying that either $3 \nmid n$, or, if $3 \mid n$, then $\delta(n)<\delta\left(\frac{n}{3}\right)$, i.e., $\|n\|<3+\left\|\frac{n}{3}\right\|$.

Let us also define:

Definition 2.23. For any real $s \geq 0$, define the set of $s$-defect numbers $A_{s}$ to be

$$
A_{s}:=\{n \in \mathbb{N}: \delta(n)<s\} .
$$

Define the set of $s$-defect leaders $B_{s}$ to be

$$
B_{r}:=\left\{n \in A_{s}: n \text { is a leader }\right\} .
$$

These sets are related by the following proposition from [3]:

Proposition 2.24. For every $n \in A_{s}$, there exists a unique $m \in B_{s}$ and $k \geq 0$ such that $n=3^{k} m$ and $\delta(n)=\delta(m)$; then $\|n\|=\|m\|+3 k$. 
Because of this, if we want to describe the set $A_{r}$, it suffices to describe the set $B_{r}$. Now we can define:

Definition 2.25. For a real number $s \geq 0$, a finite set $\mathcal{S}$ of low-defect polynomials will be called a good covering for $B_{s}$ if every $n \in B_{r}$ can be efficiently 3 -represented by some polynomial in $\mathcal{S}$ (and hence every $n \in A s r$ can be efficiently represented by some $\hat{f}$ with $f \in \mathcal{S}$ ) and if for every $f \in \mathcal{S}, \delta(f) \leq s$, with this being strict if $\operatorname{deg} f=0$.

This allows us to state the main theorem from [4:

Theorem 2.26. For any real number $s \geq 0$, there exists a good covering of $B_{s}$.

Proof. This is Theorem 4.9 from [4 rewritten in terms of Definition 2.25, and using low-defect polynomials instead of pairs. (Any low-defect pairs $(f, C)$ with $C>\|f\|$ can be filtered out of a good covering, since such a pair can never efficiently 3represent anything.)

Note that by Proposition 2.20, if $f$ is in a good covering of $B_{s}$ with leading coefficient $m$, we must have $\delta(m)+\operatorname{deg} f \leq s$.

\section{THE INTEGER DEFECT}

In this section we state some basic facts about $D(n)$, what it means, and how it may be computed.

Let us start by giving another interpretation of what $D(n)$ means:

Proposition 3.1. For a natural number n,

$$
D(n)=|\{k: n<E(k) \leq E(\|n\|)\}| .
$$

That is to say, $D(n)$ measures how far down $n$ is among numbers with complexity $\|n\|$, measured by how many values of $E$ one passes as one counts downwards towards $n$ from the largest number also having complexity $\|n\|$.

Proof. By definition, $L(n)$ is the largest $k$ such that $E(k) \leq n$. Since $E(k)$ is strictly increasing, the number of $k$ such that $n<E(k) \leq E(\|n\|)$ is equal to the difference $\|n\|-L(n)$, i.e., $D(n)$.

So for instance, one has that $D(n)=0$ if and only if $n$ is of the form $E(k)$ for some $k$, i.e., $n$ is the largest number of its complexity; while $D(n) \leq 1$ if and only if $n>E(\|n\|-1)$, i.e., $n$ is greater than all numbers of lower complexity. Numbers $n$ with $D(n) \leq 1$ will be discussed more in Section 5 .

As for properties of the integer defect, it behaves largely analogously to the real defect:

Proposition 3.2. We have:

(1) For all $n, D(n) \geq 0$.

(2) For all $n>1, L(3 n)=L(n)+3$.

(3) For $n>1$ and $k \geq 0$, one has $D\left(3^{k} n\right) \leq D(n)$, with equality if and only if $\left\|3^{k} n\right\|=3 k+\|n\|$.

(4) A number $n>1$ is stable if and only if for any $k \geq 0, D\left(3^{k} n\right)=D(n)$. 
Proof. Statement (1) is just the statement that $L(n) \leq\|n\|$; this follows from the definition of $L(n)$ as $E(\|n\|) \geq n$ and so (as $E(k)$ is increasing) one must have $L(n) \leq\|n\|$. And once statement (2) is established, statements (3) and (4) then follow from that and may be proved in exactly the same way their analogous statements in Theorem 2.1 are proved. This leaves just statement (2) to be proved. Note that, for any $k>1, E(k+3)=3 E(k)$. Therefore, for any $k>1, E(k+3) \leq 3 n$ if and only if $E(k) \leq n$, and so $L(3 n)=L(n)+3$; the only possible exception to this would be if one had $L(n)=1$, which happens only when $n=1$.

Note that while the theorem that for any $n$ there is some $k$ such that $3^{k} n$ is stable was originally proven using the defect $\delta(n)$, it could also just as well be proven using the integer defect $D(n)$.

We can also of course define a stable variant of $D(n)$ :

Definition 3.3. For a positive integer $n$, we define the stable integer defect of $n$, denoted $D_{s t}(n)$, to be $D\left(3^{k} n\right)$ for any $k$ such that $3^{k} n$ is stable.

Note that Proposition 3.2 shows that this is well-defined. We then have:

Proposition 3.4. We have:

(1) $D_{s t}(n)=\min _{k \geq 0} D\left(3^{k} n\right)$

(2) For $n>1, D_{s t}(n)=\|n\|_{s t}-L(n)$

(3) $D_{s t}(n) \leq D(n)$, with equality if and only if $n$ is stable or $n=1$

(4) For $n>1, D(n)-D_{s t}(n)=\delta(n)-\delta_{s t}(n)=\|n\|-\|n\|_{s t}$

Proof. With the exeption of (4), of which no analogue has previously been mentioned, these all follow from Proposition 3.2 and their proofs are exactly analogous to those of the statements in Proposition 2.7 meanwhile (4) follows immediately from (2) and the definition of $D(n)$.

We then also have the analogue of Proposition 3.1

Proposition 3.5. For a natural number $n>1$,

$$
D_{s t}(n)=\left|\left\{k: n<E(k) \leq E\left(\|n\|_{s t}\right)\right\}\right| .
$$

Proof. Once again, by definition, $L(n)$ is the largest $k$ such that $E(k) \leq n$. And since $E(k)$ is strictly increasing, the number of $k$ such that $n<E(k) \leq E\left(\|n\|_{s t}\right)$ is equal to the difference $\|n\|_{s t}-L(n)$, which by Proposition 3.4 is $D_{s t}(n)$.

Remark 3.6. It may seem strange that 1 needs to be excluded, given that its special status goes away when stabilized. However, $\|1\|_{s t}=0$, and $E(0)$ is not defined, so $n=1$ must still be excluded from the theorem statement.

Note, by the way:

Proposition 3.7. For any natural number $n, D(n)=0$ if and only if $D_{s t}(n)=0$.

Proof. It's immediate that a number $n$ with $D(n)=0$ is stable and so has $D_{s t}(n)=$ 0 (unless $n=1$, in which case one still has $D_{s t}(n)=0$ ). For the reverse, a number $n$ has $D_{s t}(n)=0$ if and only if there is some $k$ such that $D\left(3^{k} n\right)=0$. However, as the numbers $n$ with $D(n)=0$ are precisely those numbers of the form $3^{k}, 2 \cdot 3^{k}$, and $4 \cdot 3^{k}$, we see that if $n$ has $D_{s t}(n)=0$, it must itself be of one of these forms, and thus have $D(n)=0$. 
See Corollaries 5.2 and 5.3 for related statements.

Having discussed what $D(n)$ is and how it acts, let's finally discuss how it may be computed. The quantity $D(n)$ is just the difference $\|n\|-L(n)$. We know how to compute $\|n\|$, although not necessarily quickly; see 7 . for the currently bestknown algorithm for computing complexity, and [10] for the best-known bounds on its runtime. But the other half, computing $L(n)$, is very simple and can be done much quicker, because it's given by the following formula:

Proposition 3.8. For a natural number n,

$$
L(n)=\max \left\{3\left\lfloor\log _{3} n\right\rfloor, 3\left\lfloor\log _{3} \frac{n}{2}\right\rfloor+2,3\left\lfloor\log _{3} \frac{n}{4}\right\rfloor+4,1\right\} .
$$

Proof. The quantity $L(n)$ is by definition the largest $k$ such that $E(k) \leq n$. The largest such $k$ congruent to 0 modulo 3 is $3\left\lfloor\log _{3} n\right\rfloor$ (so long as this quantity is positive; otherwise there is none), the largest such $k$ congruent to 2 modulo 3 is $3\left\lfloor\log _{3} \frac{n}{2}\right\rfloor+2$ (with the same caveat), the largest such $k>1$ congruent to 1 modulo 3 is $3\left\lfloor\log _{3} \frac{n}{4}\right\rfloor+4$ (again with the same caveat), and of course the largest such $k$ equal to 1 is 1 . So the largest of these is $L(n)$ (and any of them that are not valid positive and thus not a valid $k$ will not affect the maximum).

Let us make here a definition that will be useful later:

Definition 3.9. For a natural number $n$, define $R(n)=\frac{n}{E(\|n\|)}$. We also define $R_{s t}(n)$ to be $R\left(3^{k} n\right)$ for any $k$ such that $3^{k} n$ is stable, or equivalently (for $n>1$ ) as $\frac{n}{E\left(\|n\|_{s t}\right)}$.

This is easily related to the defect, as was done in an earlier paper [3]:

Proposition 3.10. We have, for $n>1$,

$$
\delta(n)=\left\{\begin{array}{llll}
-3 \log _{3} R(n) & \text { if } & \|n\| \equiv 0 & (\bmod 3), \\
-3 \log _{3} R(n)+2 \delta(2) & \text { if } & \|n\| \equiv 1 & (\bmod 3), \\
-3 \log _{3} R(n)+\delta(2) & \text { if } & \|n\| \equiv 2 & (\bmod 3),
\end{array}\right.
$$

and the same relation (without the $n>1$ restriction) holds between $R_{s t}(n),\|n\|_{s t}$, and $\delta_{s t}(n)$.

Proof. The relation between $R(n)$ and $\delta(n)$ is just Proposition A.3 from [3] and the proof for the stable case is exactly analogous.

Now we see that in addition to being easy to compute $L(n)$, it's also simple to determine $D(n)$ from $\delta(n)$, at least if we know the value of $\|n\|$ modulo 3 , which technically is implicit in $\delta(n)$. First, a definition:

Definition 3.11. Let $a$ be a congruence class modulo 3 and $k$ be a whole number. Define

$$
t_{a}(k)= \begin{cases}k & \text { if } k \equiv a \quad(\bmod 3) \\ k+\delta(2) & \text { if } k \equiv a+1 \quad(\bmod 3) \\ k+2 \delta(2) & \text { if } k \equiv a+2 \quad(\bmod 3)\end{cases}
$$

Now:

Theorem 3.12. Let $n>1$ be a natural number. Then $D(n)$ is equal to the smallest $k$ such that $\delta(n) \leq t_{\|n\|}(k)$. Moreover, if $n$ is any natural number, $D_{\text {st }}(n)$ is equal to the smallest $k$ such that $\delta_{s t}(n) \leq t_{\|n\|_{s t}}(k)$. 
Since two numbers with the same defect also have the same complexity modulo $3($ and $\delta(n)=1$ if and only if $n=1)$, and the analogous statement is also true of stable complexity and defect, in particular we have that if $\delta(n)=\delta(m)$ then $D(n)=D(m)$, and if $\delta_{s t}(n)=\delta_{s t}(m)$ then $D_{s t}(n)=D_{s t}(m)$.

Note in addition that since $\delta(n)=\delta(m)$ implies $\delta_{s t}(n)=\delta_{s t}(m)$ (see statement (2) in Proposition 2.7) one has that if $\delta(n)=\delta(m)$ then $D_{s t}(n)=D_{s t}(m)$.

Theorem 3.12 makes precise how $D(n)$ is "almost $\lceil\delta(n)\rceil$ ". It is, as was noted in the introduction, not the same, but it is the smallest $k$ such that $\delta(n) \leq t_{\|n\|}(k)$, where $t_{\|n\|}(k)$ may not be exactly $k$ but never differs from it by more than $2 \delta(2)<$ 0.215 .

Proof. We prove only the non-stabilized case as the stabilized case is exactly analogous. We assume $n>1$.

From Proposition 3.1, we can see that $D(n)$ is determined by $R(n)$ and the value of $\|n\|$ modulo 3. Specifically,

$$
D(n)=\left|\left\{k: R(n)<\frac{E(k)}{E(\|n\|)} \leq 1\right\}\right|,
$$

so $D(n)$ is the number of values of $\frac{E(k)}{E(\|n\|)}$ in $(R(n), 1]$. What are the values of this? They can be obtained as products of values $\frac{E(k)}{E(k+1)}$; this is equal to $2 / 3$ when $k \equiv 1$ or $2(\bmod 3)($ for $k>1)$ and to $3 / 4$ when $k \equiv 0(\bmod 3)$.

Thus, if $\|n\| \equiv 0(\bmod 3), D(n)$ will increase whenever $R(n)$ passes a value of the sequence $1, \frac{2}{3}, \frac{4}{9}, \frac{1}{3}, \frac{2}{9}, \frac{4}{27}, \frac{1}{9}, \ldots$; if $\|n\| \equiv 1(\bmod 3)$, whenever it passes a value of the sequence $1, \frac{3}{4}, \frac{1}{2}, \frac{1}{3}, \frac{1}{4}, \frac{1}{6}, \frac{1}{9}, \ldots$; and if $\|n\| \equiv 2(\bmod 3)$, whenever it passes a value of the sequence $1, \frac{2}{3}, \frac{1}{2}, \frac{1}{3}, \frac{2}{9}, \frac{1}{6}, \frac{1}{9}, \ldots$ (These sequences are just the sequences obtained by taking products of one of the three shifts of the periodic sequence $\frac{2}{3}, \frac{2}{3}, \frac{3}{4}, \frac{2}{3}, \frac{2}{3}, \frac{3}{4} \ldots$; note that regardless of which shift is used, the repeating part of the sequence always has a product of $\frac{1}{3}$, and so the product sequences will always consist of three interwoven geometric sequences each with ratio $\frac{1}{3}$.)

It just remains, then, to convert these values of $R(n)$ to their equivalents in defects, which can be done with Proposition 3.10. Once this is done one finds that the values of $\delta(n)$ where $D(n)$ increases are precisely those listed in the definition of $t_{\|n\|}$, which completes the proof.

Theorem 3.12 will form half the proof of Theorem 1.8, and its stable analogue, Theorem 4.2 it tells us that the values of $D(n)$ "switch over" when $\delta(n)$ is of the form $k, k+\delta(2)$, or $k+2 \delta(2)$ depending on the congruence class of $k-\|n\|$ modulo 3. The other half the proof is, of course, Theorem 1.9 (and its stable analogue, Theorem 4.1), which will tell us that these changeover points are exactly the limits of the initial $\omega^{k}$ defects in $\mathscr{D}^{a}$ (or $\left.\mathscr{D}_{s t}^{a}\right)$.

\section{THE ORDER INTERPRETATION OF $D(n)$}

In this section we aim to prove Theorem [1.9] using the methods described in Section 1.3. combined with Theorem 3.12 from the previous section, this will prove Theorem 1.8. Really, we want to prove generalizations:

Theorem 4.1. For any $k \geq 0$ and a a congruence class modulo 3 , the order type of $\mathscr{D}^{a} \cap\left[0, t_{a}(k)\right]$ and the order type of $\mathscr{D}_{s t}^{a} \cap\left[0, t_{a}(k)\right]$ are both equal to $\omega^{k}$. 
Theorem 4.2. Let $n>1$ be a natural number. Let $\zeta$ be the order type of $\mathscr{D}\|n\| \cap$ $[0, \delta(n))$. Then $D(n)$ is equal to the smallest $k$ such that $\zeta<\omega^{k}$. The same is true if we replace $\delta(n)$ by $\delta_{s t}(n), \mathscr{D}^{\|n\|}$ by $\mathscr{D}_{s t}^{\|n\|_{s t}}$, and $D(n)$ by $D(n)_{s t}$.

Note that the proofs in this section will rely heavily on the results in Sections 2.2 and 2.3. Before we prove these, though, we will need a slight elaboration on Proposition 2.19.

Proposition 4.3. Let $f$ be a low-defect polynomial of degree $d$ with $\delta(f)<d+1$. Then the order type of the set of all $\delta(N)$ for $n 3$-represented by $\hat{f}$ is exactly $\omega^{d}$.

Proof. By Proposition 2.19, $\left\{\delta\left(\hat{f}\left(3^{n_{1}}, \ldots, 3^{n_{d}}\right)\right):\left(n_{1}, \ldots, n_{d}\right) \in S\right\}$ has order type less than $\omega^{d}$. Meanwhile, also by Proposition 2.19, the set

$$
\left\{\delta\left(f\left(3^{n_{1}}, \ldots, 3^{n_{d}}\right)\right):\left(n_{1}, \ldots, n_{d}\right) \notin S\right\}
$$

has order type at least $\omega^{d}$, and is cofinal in $[0, \delta(f))$ (or $[0, \delta(f)]$ if $\operatorname{deg} f=0$ ) and therefore in the set of all $\delta(N)$ for $n$ 3-represented by $\hat{f}$. But in fact, for $\left(n_{1}, \ldots, n_{d}\right) \notin S$, one has $\delta\left(\hat{f}\left(3^{n_{1}}, \ldots, 3^{n_{d+1}}\right)=\delta_{f}\left(n_{1}, \ldots, n_{d}\right)\right.$, and so this set (even when $f\left(3^{n_{1}}, \ldots, 3^{n_{d}}\right)$ is replaced by $\left.\hat{f}\left(3^{n_{1}}, \ldots, 3^{n_{d+1}}\right)\right)$ is a subset of the image of $\delta_{f}$, which by Proposition 2.16 has order type $\omega^{d}$. So the conditions of Proposition 2.18 apply, and the union of these two sets, the set of all $\delta(n)$ for $N$ 3-represented by $\hat{f}$, has order type at most $\omega^{d}$. We already know by Proposition 2.16 it has order type at least $\omega^{d}$, so this proves the claim.

We now prove the main theorems of this section.

Proof of Theorem 4.1. We need to show that the order type of $\mathscr{D}^{a} \cap\left[0, t_{a}(k)\right]$, as well as the order type of $\mathscr{D}_{s t}^{a} \cap\left[0, t_{a}(k)\right]$, are both equal to $\omega^{k}$. This proof breaks down into two parts, an upper bound and a lower bound. Since $\mathscr{D}_{s t}^{a} \subseteq \mathscr{D}^{a}$, it suffices to prove the upper bound for $\mathscr{D}^{a} \cap\left[0, t_{a}(k)\right]$, and the lower bound for $\mathscr{D}_{s t}^{a} \cap\left[0, t_{a}(k)\right]$.

We begin with the upper bound. First, we observe that $t_{a}(k)$ is not itself an element of $\mathscr{D}^{a}$ for any $k>0$. We can see this as neither $k+\delta(2)$ nor $k+2 \delta(2)$ is a defect for any $k>0$ (such a defect would have to come from some number $n$ satisfying $3^{\ell} n=2$ or $3^{\ell} n=4$ for $\ell>0$, which is impossible), and similarly no nonzero integer is a defect except $k=1$, which though an element of $\mathscr{D}$ is by definition excluded from all three $\mathscr{D}^{a}$. Thus $\mathscr{D}^{a} \cap\left[0, t_{a}(k)\right]=\mathscr{D}^{a} \cap\left[0, t_{a}(k)\right)$ and we may concern ourselves with the order type of the latter.

Now we take a good covering $\mathcal{S}$ of $B_{t_{a}(k)}$ as per Theorem 2.26. For any $f \in \mathcal{S}$ with leading coefficient $m$, we have the inequality $\delta(m)+\operatorname{deg} f \leq \delta(f) \leq t_{a}(k)$. In particular, for any $f \in \mathcal{S}$, we have $\operatorname{deg} f \leq\left\lfloor t_{a}(k)\right\rfloor=k$.

Suppose now that $\operatorname{deg} f=k$; then there is more we can say. For in this case, we have $\delta(m) \leq t_{a}(k)-k \leq 2 \delta(2)$. Thus $\delta(m) \in\{0, \delta(2), 2 \delta(2)\}$ by Proposition 2.8 Note that by their respective definitions, $\delta(f) \equiv \delta(m)(\bmod 1)$; and, as noted above, $\delta(f) \geq \operatorname{deg} f=k$, and so $\delta(f)=k+\delta(m) \in\{k, k+\delta(2), k+2 \delta(2)\}$. Note that $\delta(f)=k+\delta(m)$ means that

$$
k+\|m\|-3 \log _{3} m=\|f\|-3 \log _{3} m
$$

and therefore $\|f\|=k-\|m\|$. Moreover, if $\delta(m)=0$, then $m$ is of the form $3^{\ell}$ (for some $\ell>0$ ) and $\|m\|=3 \ell$, if $\delta(m)=\delta(2)$ then $m$ is of the form $2 \cdot 3^{\ell}$ with 
$\|m\|=2+3 \ell$, and if $\delta(m)=2 \delta(2)$ then $m$ is of the form $4 \cdot 3^{\ell}$ with $\|m\|=4+3 \ell$; from this we can conclude that, modulo 3 ,

$$
\|f\| \equiv \begin{cases}k & \text { if } \delta(f)=k \\ k-2 & \text { if } \delta(f)=k+\delta(2) \\ k-1 & \text { if } \delta(f)=k+2 \delta(2)\end{cases}
$$

Now, let $T_{f}=\left\{\delta\left(\hat{f}\left(3^{n_{1}}, \ldots, 3^{n_{d+1}}\right)\right): n_{1}, \ldots, n_{d+1} \geq 0\right\} \cap \mathscr{D}^{a}$, where $d=\operatorname{deg} f$. Then by the assumption that $\mathcal{S}$ is a good covering of $B_{t_{a}(k)}$, we have that

$$
\mathscr{D}^{a} \cap\left[0, t_{a}(k)\right)=\bigcup_{f \in \mathcal{S}} T_{f} .
$$

We want to show that the conditions of Proposition 2.18 hold for the sets $T_{f}$, so that we can conclude that $\mathscr{D}^{a} \cap\left[0, t_{a}(k)\right)$ has order type at most $\omega^{k}$. If $\operatorname{deg} f<$ $k$, then, by Proposition 2.16. $T_{f}$ has order type less than $\omega^{k}$, and thus so does $T_{f} \cap \mathscr{D}^{a}$. Meanwhile, if $\operatorname{deg} f=k$, then since $\delta(f) \leq t_{a}(k)<k+1$, we can apply Proposition 4.3 to conclude that the set of $\delta(N)$ for $N$ 3-represented by $\hat{f}$ has order type $\omega^{k}$. However, if $\delta(f) \neq t_{a}(k)$, then by the previous paragraph and Proposition 2.19, we see that while this has order type $\omega^{k}, T_{f}$, which is its intersection with $\mathscr{D}^{a}$, has order type less than $\omega^{k}$.

It remains to check, then, that when $\operatorname{deg} f=k$ and $\delta(f)=t_{a}(k)$, that the set $T_{f}$ is cofinal in $\bigcup_{f \in \mathcal{S}} T_{f}=\mathscr{D}^{a} \cap\left[0, t_{a}(k)\right)$, or in other words, just that it's cofinal in $\left[0, t_{a}(k)\right)$. But this follows from Proposition 2.19, which in fact goes further and states that $T_{f} \cap \mathscr{D}_{s t}^{a}$ is cofinal in $[0, \delta(f))=\left[0, t_{a}(k)\right)$.

Thus, applying Proposition 2.18, we conclude that $\mathscr{D}^{a} \cap\left[0, t_{a}(k)\right)$ has order type at most $\omega^{k}$. This proves the upper bound.

To prove the lower bound, let's consider the low-defect polynomial

$$
f=\left(\cdots\left(\left(m x_{1}+1\right) x_{2}+1\right) \cdots\right) x_{k}+1
$$

(for a particular $m$ to be chosen shortly) which has $\|f\|=\|m\|+k$. (The upper bound on $\|f\|$ is immediate and the lower bound follows from Proposition 2.20.) For the value of $m$, we take

$$
m= \begin{cases}3 & \text { if } k-a \equiv 0 \quad(\bmod 3) \\ 4 & \text { if } k-a \equiv 2 \quad(\bmod 3) \\ 2 & \text { if } k-a \equiv 1 \quad(\bmod 3)\end{cases}
$$

so that $\|m\| \equiv a-k(\bmod 3)$ and $\|f\| \equiv a(\bmod 3)$, meaning $\mathscr{D}_{s t}^{\|f\|}=\mathscr{D}_{s t}^{a}$.

Then $\delta(f)=t_{a}(k)$ and so in particular $\delta(f)<k+1$, meaning once again we can apply Proposition 2.19 to conclude that the set

$$
\left\{\delta\left(f\left(3^{n_{1}}, \ldots, 3^{n_{k}}\right)\right):\left(n_{1}, \ldots, n_{k}\right) \in \mathbb{Z}_{\geq 0}^{k}\right\} \cap \mathscr{D}_{s t}^{\|f\|}
$$

has order type at least $\omega^{k}$. Since this set is bounded above by $\delta(f)=t_{a}(k)$ and $\mathscr{D}_{s t}^{\|f\|}=\mathscr{D}_{s t}^{a}$, we conclude that the order type of $\mathscr{D}_{s t}^{a} \cap\left[0, t_{a}(k)\right)$ is at least $\omega^{k}$. This completes the proof.

In particular this encompasses Theorem 1.9 .

Proof of Theorem 1.9. This is just a rephrasing of Theorem4.1 with the application to $\mathscr{D}_{s t}^{a}$ omitted. 
Having proven Theorem 4.1, we can now combine it with Theorem 3.12 to obtain Theorem 4.2 and Theorem 1.8 :

Proof of Theorem 4.2. By Theorem 3.12, $D(n)$ is equal to the smallest $k$ such that $\delta(n) \leq t_{\|n\|}(k)$. However, since the order type of $\mathscr{D}\|n\| \cap\left[0, t_{\|n\|}(k)\right)$ is equal to $\omega^{k}$, one has that $\zeta<\omega^{k}$ if and only if $\delta(n)<t_{\|n\|}(k)$. Thus $D(n)$ is equal to the smallest $k$ such that $\zeta<\omega^{k}$. The proof for the stabilized version is similar.

Proof of Theorem 1.8, This is just the special case of Theorem 4.2 where we only consider $\delta(n)$ and not $\delta_{s t}(n)$.

\section{Numbers $n$ With $D(n) \leq 1$}

In the previous section we showed that the numbers with integral defect at most $k$ correspond to the initial $\omega^{k}$ defects in each of $\mathscr{D}^{0}, \mathscr{D}^{1}$, and $\mathscr{D}^{2}$. In this section we take a closer look at the initial $\omega$, the numbers with integral defect at most 1 , and use this to generalize Theorem 1.10 .

Let's start by listing all the numbers with integral defect at most 1:

Theorem 5.1. A natural number $n$ satisfies $D(n) \leq 1$ if and only if it can be written in one of the following forms:

(1) 1 , of complexity 1

(2) $2^{a} 3^{k}$ for $a \leq 10$, of complexity $2 a+3 k$ (for $a, k$ not both zero)

(3) $2^{a}\left(2^{b} 3^{\ell}+1\right) 3^{k}$ for $a+b \leq 2$, of complexity $2(a+b)+3(\ell+k)+1$ (for $b, \ell$ not both zero).

Proof. By Theorem 3.12, any $n$ with $D(n) \leq 1$ must have $\delta(n) \leq 1+2 \delta(2)$. Theorem 31 from [6] gives a classification of all numbers $n$ with $\delta(n)<12 \delta(2)$, together with their complexities; since $12 \delta(2)>1+2 \delta(2)$, any $n$ with $D(n) \leq 1$ may be found among these. (One may also use the algorithms from 2 to find such a classification.) It is then a straightforward matter to determine which of the $n$ listed there have $D(n) \leq 1$.

This has an important corollary:

Corollary 5.2. For any natural number $n, D(n)=1$ if and only if $D_{s t}(n)=1$.

Proof. From Theorem [5.1, we see that if $D(n) \leq 1$ then we also have $D\left(3^{k} n\right) \leq 1$, and if $D\left(3^{k} n\right) \leq 1$ then we have $D(n) \leq 1$; this shows that $D(n) \leq 1$ if and only if $D_{s t}(n) \leq 1$. Combining this with Proposition 3.7 proves the claim.

From this we can conclude:

Corollary 5.3. For any natural number $n>1$, if $D(n) \leq 2$ then $n$ is stable (and so $\left.D_{s t}(n) \leq 2\right)$.

Proof. If $D(n)=0$ or $D(n)=1$, this is Proposition 3.7 or Corollary 5.2, respectively. If $D(n)=2$, then for any $k \geq 0$, if we had $D\left(3^{k} n\right)<2$, then, by Proposition 3.7 and Corollary 5.2 , we would have $D(n)<2$, contrary to assumption; thus $D\left(3^{k} n\right)=2$ for all $k \geq 0$, i.e., $n$ is stable (by Proposition 3.4).

Note that the converse, that if $D_{s t}(n) \leq 2$ then $D(n) \leq 2$, does not hold; for instance, we can consider 107 , which has $D_{s t}(107)=2$ but $D(107)=3$, or 683 , which has $D_{s t}(683)=2$ but $D(683)=4$. (It is easy to verify that these numbers 
have stable integer defect at most 2 because $D(321)=D(2049)=2$; that these numbers do then have stable integer defect equal to 2 and not any lower can then be inferred from Corollary 5.3 . Alternately, the stable complexity, and thus stable integer defect, may be computed with the algorithms from [2].)

However, for our purposes, the most important consequence of Corollary 5.2 is the following rephrasing of it:

Proposition 5.4. Let $k>1$ be a natural number and suppose $h$ is a value of $R$ corresponding to a defect in the initial $\omega$ of $\mathscr{D}^{k}$. Then if $h E(k)$ is a natural number $n$, one has $\|n\|=k$, and, moreover, $n>E(k-1)$.

Proof. Suppose $h E(k)$ is a natural number $n$. We must have $n>1$ because having $h=1 / E(k)$ for $k>1$ would by Proposition 3.10 correspond to a defect which is a nonzero integer, and these (by Proposition 2.7) do not exist.

Then there is, by defintion of $h$, some number $m>1$ with $\|m\| \equiv k(\bmod 3)$ and $R(m)=h$, i.e., $m=h E(\|m\|)$. Since $\|m\| \equiv k(\bmod 3)$ we see that $m=n 3^{\ell}$ for some $\ell \in \mathbb{Z}$, where $\ell=\frac{\|m\|-k}{3}$. But also we have $D(m) \leq 1$. Therefore, whether $\ell \geq 0$ or $\ell \leq 0$, we must have $D_{s t}(n) \leq 1$, and so, by Proposition 3.7 and Corollary 5.2 , we have $D(n) \leq 1$. Then by Proposition 3.2, we have $\|m\|=\|n\|+3 \ell$. From the definition of $\ell$ we also have $\|m\|=k+3 \ell$ and thus we conclude that $\|n\|=k$. And since $D(n)=1$ this means (by Proposition 3.1) that $n>E(k-1)$.

We can now prove Theorem 1.12 .

Proof of Theorem 1.12, Suppose we want to determine the $r$ 'th largest number of complexity $k$. This is equivalent to determining the $r$ 'th largest value of $R(n)=$ $\frac{n}{E(k)}$ that occurs among numbers $n$ of complexity $k$, which is equivalent to determining the $r$ 'th smallest defect $\delta(n)$ that occurs among numbers $n$ of complexity $k$.

Now, we can easily determine the initial values $\alpha_{0}, \ldots, \alpha_{r}$ of $\mathscr{D}^{k}$; let $h_{0}, \ldots, h_{r}$ be the corresponding values of the function $R$, as given by Proposition 3.10. (For instance, for a way of getting $h_{0}, \ldots, h_{r}$ directly rather than going by means of defects, one may take the numbers $n$ given in Theorem 5.1, group them by the residues of $\|n\|$ modulo 3 , then sort them in decreasing order by $R(n)$; note that the values of $R(n)$ obtained this way for any one congruence class of $\|n\|$ modulo 3 will have reverse order type $\omega$.) One may see Tables [4 [5] and 6 for tables of the resulting values of $h$. Then certainly, the $r$ 'th largest number of complexity $k$ is at most $h_{r} E(k)$, because the set of values of $R(n)$ occuring for $n$ with $\|n\|=k$ is a subset of the values of $R(n)$ occuring for $n>1$ with $\|n\| \equiv k(\bmod 3)$. However, it will only be exactly the $r$ 'th largest number of complexity $k$ if all of $h_{1}$ through $h_{r}$ do indeed occur for some $n$ with $\|n\|=k$.

But, by Proposition 5.4, this is equivalent to just requiring that all of the numbers $h_{0} E(k), \ldots, h_{r} E(k)$ are indeed whole numbers (and moreover when this does occur one will have $\left.h_{i} E(k)>E(k-1)\right)$. In other words, this is the same as requiring

$$
k \geq\left\{\begin{array}{lll}
-3 \min _{s \leq r} v_{3}\left(h_{s}\right) & \text { if } k \equiv 0 & (\bmod 3) \\
-3 \min _{s \leq r} v_{3}\left(h_{s}\right)+4 & \text { if } k \equiv 1 & (\bmod 3) \\
-3 \min _{s \leq r} v_{3}\left(h_{s}\right)+2 & \text { if } k \equiv 2 & (\bmod 3) .
\end{array}\right.
$$

So we have our $h_{r, a}$, and we can take $K_{r, a}$ to be given by this formula. (Although since for $K_{0,0}$ it may not may make much sense to take $K_{0,0}=0$, one may wish to take $K_{0,0}=3$ instead, as we have done in Table 1.) 
Combining this with Tables 4, 5, and 6yields Tables 1, 2, and 3, and proves the theorem.

Remark 5.5. While in the proof of Theorem 1.12 we have referred to facts proved in Section 4, none of the techniques deployed in that section are necessary for the proof. For instance, one can easily verify the values of the $\overline{\mathscr{D}^{a}}(\omega)$ by directly determining the initial $\omega$ elements without needing to determine it for all $\omega^{k}$; indeed Tables 4, 5, and 6 essentially do this directly from Theorem 5.1.

TABLE 4. Table of $h_{r}$ for $k \equiv 0(\bmod 3)$.

\begin{tabular}{|c|c|c|}
\hline r & $h$ & Corresponding leader \\
\hline $\bar{c}$ & 1 & $3=3^{1} 2^{0}=2^{1} 3^{0}+1$ \\
\hline 1 & $8 / 9$ & $8=2^{3}=2^{1}\left(3^{1}+1\right)$ \\
\hline 2 & $64 / 81$ & $64=2^{6}$ \\
\hline 3 & $7 / 9$ & $7=2^{1} 3^{1}+1$ \\
\hline 4 & $20 / 27$ & $20=2^{1}\left(3^{2}+1\right)$ \\
\hline 5 & $19 / 27$ & $19=2^{1} 3^{2}+1$ \\
\hline 6 & $512 / 729$ & $512=2^{9}$ \\
\hline$($ for $n \geq 4) \quad 2 n-1$ & $2 / 3+2 / 3^{n}$ & $2^{1}\left(3^{n-1}+1\right)$ \\
\hline$($ for $n \geq 4) \quad 2 n$ & $2 / 3+1 / 3^{n}$ & $2^{1} 3^{n-1}+1$ \\
\hline
\end{tabular}

TABLE 5. Table of $h_{r}$ for $k \equiv 2(\bmod 3)$.

\begin{tabular}{|rr|r|r|}
\hline$r$ & $h$ & Corresponding leader \\
\hline & 0 & 1 & $2=2^{1}$ \\
& 1 & $8 / 9$ & $16=2^{4}=2^{2}\left(3^{1}+1\right)$ \\
& 2 & $5 / 6$ & $5=2^{2} 3^{0}+1$ \\
& 3 & $128=2^{7}$ \\
& 4 & $64 / 81$ & $7 / 9$ \\
& 5 & $20 / 27$ & $14=2^{1}\left(2^{1} 3^{1}+1\right)$ \\
& 6 & $13 / 18$ & $40=2^{2}\left(3^{2}+1\right)$ \\
& 7 & $13=2^{2} 3^{1}+1$ \\
& 8 & $512 / 729$ & $38=2^{1}\left(2^{1} 3^{2}+1\right)$ \\
$($ for $n \geq 4)$ & $3 n-3$ & $2 / 3+2 / 3^{n}$ & $2^{2}\left(3^{n-1}+1\right)$ \\
$($ for $n \geq 4)$ & $3 n-2$ & $2 / 3+1 /\left(2 \cdot 3^{n-1}\right)$ & $2^{2} 3^{n-1}+1$ \\
$($ for $n \geq 4)$ & $3 n-1$ & $2 / 3+1 / 3^{n}$ & $2^{1}\left(2^{1} 3^{n-1}+1\right)$ \\
\hline
\end{tabular}

TABLE 6 . Table of $h_{r}$ for $k \equiv 1(\bmod 3)$ with $k>1$.

\begin{tabular}{|c|c|c|c|}
\hline & $r$ & $h$ & Corresponding leader \\
\hline & $\overline{0}$ & 1 & $4=2^{2}=3^{1}+1$ \\
\hline & 1 & $8 / 9$ & $32=2^{5}$ \\
\hline & 2 & $5 / 6$ & $10=3^{2}+1$ \\
\hline & 3 & $64 / 81$ & $256=2^{8}$ \\
\hline (for $n \geq 2$ ) & $n+2$ & $3 / 4+1 /\left(4 \cdot 3^{n}\right)$ & $3^{n+1}+1$ \\
\hline
\end{tabular}


As a final note, it is worth making formal a statement mentioned in Section 1.2 that the numbers $h E(k)$ coming from Theorem 1.12 are almost exactly the $n$ with $D(n) \leq 1$ :

Proposition 5.6. A number $n$ has $D(n) \leq 1$ if and only if there are some $\ell \geq 0$, $k \geq 1$, and $r \geq 0$ such that $k \geq K_{r, k}$ and $3^{\ell} n=h_{r, k} E(k)$.

Proof. We already know that if $k \geq K_{r, k}$ then, if we let $m=h_{r, k} E(k)$, that $m>E(k-1)=E(\|m\|-1)$, i.e., $D(m) \leq 1$, and so if $m=3^{\ell} n$, then $D(n) \leq 1$ by Corollary 5.2 ,

Conversely, if $D(n) \leq 1$, let $h=R(n)$; then by the construction of the $h_{r, a}$ in the proof of Theorem 1.12, and the fact that the values of $R(n)$ for numbers $n$ with $\|n\|$ in a fixed congruence class modulo 3 have reverse order type $\omega$, there is some $r$ such that $h=h_{r,\|n\|}$. We may then take any $k \geq K_{r,\|n\|}$ with $k \equiv\|n\|(\bmod 3)$; then $3^{\ell} n=h_{r,\|n\|} E(k)=h_{r, k} E(k)$ for $\ell=\frac{k-\|n\|}{3}$.

Acknowledgements. Work of the author was supported by NSF grants DMS0943832 and DMS-1101373.

\section{REFERENCES}

[1] H. Altman, Internal Structure of Addition Chains: Well-Ordering, Theoretical Computer Science (2017), doi:10.1016/j.tcs.2017.12.002

[2] H. Altman, Integer Complexity: Agorithms and Computational Results, arXiv:1606.03635, 2016

[3] H. Altman, Integer Complexity and Well-Ordering, Michigan Mathematical Journal 64 (2015), no. 3, 509-538.

[4] H. Altman, Integer Complexity: Representing Numbers of Bounded Defect, Theoretical Computer Science 652 (2016), 64-85.

[5] H. Altman and J. Arias de Reyna, Integer Complexity, Stability, and Self-Similarity, in preparation

[6] H. Altman and J. Zelinsky, Numbers with Integer Complexity Close to the Lower Bound, Integers 12 (2012), no. 6, 1093-1125.

[7] J. Arias de Reyna and J. Van de Lune, Algorithms for determining integer complexity, arXiv:1404.2183, 2014

[8] A. Brauer, On Addition Chains, Bull. Amer. Math. Soc. 45 (1939), 736-739.

[9] P. W. Carruth, Arithmetic of ordinals with applications to the theory of ordered abelian groups, Bull. Amer. Math. Soc. 48 (1942), 262-271.

[10] K. Cordwell, A. Epstein, A. Hemmady, S. J. Miller, E. A. Palsson, A. Sharma, S. Steinerberger, Y. N. Truong Vu, On algorithms to calculate integer complexity, arXiv:1706.08424, 2017

[11] D. H. J. De Jongh and R. Parikh, Well-partial orderings and hierarchies, Indag. Math. 39 (1977), 195-206.

[12] H. Dellac, Interméd. Math. 1 (1894), 162-164.

[13] A. A. Gioia, M. V. Subbarao, and M. Sugunamma, The Scholz-Brauer Problem in Addition Chains, Duke Math. J. 29 (1962), 481-487.

[14] R. K. Guy, Some suspiciously simple sequences, Amer. Math. Monthly, 93 (1986), 186-190; and see 94 (1987), $965 \& 96$ (1989), 905.

[15] R. K. Guy, Unsolved Problems in Number Theory, Third Edition, Springer-Verlag, New York, 2004, pp. 399-400.

[16] D. E. Knuth, The Art of Computer Programming, Vol. 2, Third Edition, Addison-Wesley, Reading, Massachusetts, pp. 461-485

[17] K. Mahler and J. Popken, On a maximum problem in arithmetic (Dutch), Nieuw Arch. Wiskunde, (3) 1 (1953), 1-15; MR 14, 852e.

[18] D. A. Rawsthorne, How many 1's are needed?, Fibonacci Quart. 27 (1989), 14-17; MR 90b:11008. 
[19] A. Scholz, Aufgabe 253, Jahresbericht der Deutschen Mathematikervereinigung, Vol. 47, Teil II, B. G. Teubner, Leipzig and Berlin, 1937, pp. 41-42.

[20] M. V. Subbarao, Addition Chains - Some Results and Problems, Number Theory and Applications, Editor R. A. Mollin, NATO Advanced Science Series: Series C, V. 265, Kluwer Academic Publisher Group, 1989, pp. 555-574.

[21] I. Volkovich, Characterizing Arithmetic Read-Once Formulae, ACM Trans. Comput. Theory 8 (2015), no. 1, Art. 2, 19 pp.

[22] J. Zelinsky, An Upper Bound on Integer Complexity, in preparation 